\title{
Estresse em Pacientes com Glaucoma Primário de Ângulo Aberto ${ }^{1}$
}

\author{
Maira Santos Marinho Moura \\ Universidade de São Paulo \\ Karina Franco Zihlmann2 \\ Universidade Nove de Julho \\ Maria Lívia Tourinho Moretto \\ Newton Kara- José \\ Remo Susanna Junior \\ Mara Cristina Souza de Lucia \\ Universidade de São Paulo
}

\begin{abstract}
RESUMO - Glaucoma Primário de Ângulo Aberto (GPAA) é uma importante causa de cegueira no mundo. O presente trabalho teve como objetivo investigar: (1) presença e tipo de estresse; (2) relação do número de colírios e estresse; (3) percepção do glaucoma e tratamento. Um estudo transversal e quantitativo foi realizado com 102 pacientes do Ambulatório de Oftalmologia do HC-FMUSP, com roteiro temático e Inventário de Sintomas de Estresse de Lipp. A maioria dos pacientes apresentou estresse $(65,7 \%)$ e não houve correlação entre estresse e número de colírios. "Tempo de tratamento", "dificuldades na vida diária" e "dificuldades em pingar o colírio" foram variáveis independentemente associadas ao estresse. Conclui-se que o estresse pode interferir negativamente no enfrentamento da doença em pacientes com GPAA.
\end{abstract}

Palavras-chave: estresse; psicologia hospitalar; glaucoma; adesão ao tratamento.

\section{Stress in Patients with Primary Open-Angle Glaucoma}

\begin{abstract}
Primary Open-Angle Glaucoma (POAG) is an important cause of blindness worldwide. This paper aimed to investigate: (a) the presence and type of stress; (b) the relationship between the number of eye-drops and stress; (c) perceptions regarding glaucoma and its treatment. In a transversal and quantitative study, we assessed 102 patients from the Ophthalmology Clinic of the HC-FMUSP by means of a thematic interview and the Lipp's Stress Symptoms Inventory (LSSI). Most of the patients were stressed $(65,7 \%)$ and there was no correlation between stress and the number of eye-drops. "Time of treatment", "difficulties in daily life" and "difficulties to instillate the drops" were independently associated with stress. In conclusion, stress can negatively interfere in coping with POAG.
\end{abstract}

Keywords: stress; hospital psychology; glaucoma; adherence to treatment.

O glaucoma é uma doença crônica e uma das maiores causas de cegueira no mundo (Susanna Jr. \& Weinreb, 2005). Porém, há poucos estudos sobre os aspectos emocionais dos pacientes acometidos por essa doença. Neste estudo, foi investigada a presença de estresse entre pacientes com Glaucoma Primário de Ângulo Aberto (GPAA), pois se trata de uma forma bastante comum de glaucoma entre pessoas de ambos os sexos, com idade acima de 50 anos (Flammer, 2001), com significativo risco de evolução para cegueira (Deokule, Sadiq \& Shah, 2004).

O glaucoma é uma neuropatia óptica progressiva com dano estrutural, frequentemente acompanhado por um tipo específico de defeito de campo visual (Susanna Jr. \& Weinreb, 2005). Trata-se de uma doença insidiosa e assintomática em seus estágios iniciais (Wilensky, 1996). Portanto, pacientes com histórico de glaucoma na família devem submeter-se a acompanhamento médico periódico, a partir dos 40 anos de idade (Gabelt \& Kaufman, 2005), pois o aumento da Pressão

1 Agradecemos à Fundap, pela Bolsa de Aprimoramento concedida à primeira autora durante a elaboração deste trabalho.

2 Endereço para correspondência: Rua Lisboa, 518 Apto 94, Jardim América. São Paulo, SP. CEP 05413-001.E-mail: zihlmann@usp.br.
Intra-Ocular (PIO) não está, necessariamente, associado a sintomas específicos (Flammer, 2001).

$\mathrm{O}$ uso de medicamentos, na forma de colírios, é o tratamento de escolha para controle do glaucoma. Nos últimos anos, a intervenção cirúrgica vem sendo postergada, sendo necessária somente em casos em que as medicações tornaram-se ineficazes para o controle da PIO (Azura-Blanco, 2002). O tratamento do glaucoma tem alto custo, não somente para os cofres públicos, como também para o próprio indivíduo, quando o mesmo compra a medicação ou tem que se submeter a intervenções cirúrgicas (Silva, Vasconcellos, Temporim, Costa \& Kara-José, 2002).

Podemos observar que o tratamento do glaucoma pode trazer mudanças no cotidiano do sujeito, já que o uso contínuo do colírio torna-se uma tarefa complexa (Winfield, Jessiman, Williams \& Esakowitz, 1990). Essa questão, também, pode influenciar na adesão ao tratamento no longo prazo, como comentam Demailly, Zoute e Castro (1989), que observaram maior adesão somente no início do tratamento. Além disso, o apoio familiar na ajuda da instilação dos colírios aparece como um fator positivo em relação à adesão ao tratamento (Winfield \& cols., 1990). 
Por sua vez, trabalhos de Demailly e cols. (1989), Lim e cols. (2007) e Spinelli, Faroni e Castellini (1998) referem que mudanças na vida diária de pacientes com glaucoma, como, por exemplo, o problema da diminuição ou da perda de autonomia, em função da perda da visão, tendem a levá-los a lidarem com suas limitações de forma defensiva, para evitarem situações conflitantes.

Vários estudos apontam prejuízos significativos na qualidade de vida dos pacientes com glaucoma, devido à dificuldade visual e ao tipo de tratamento (Balkrishnan, Bond, Byerly, Camanho \& Anderson, 2003; Perfetti, Varotto, Massagrandi, Pagliani \& Bonomi, 1998; Spaeth, Walt \& Kenner, 2006; Vaidergorn \& cols., 2003).

Além de entender o prejuízo causado na qualidade de vida, torna-se de interesse também entender se o estresse está presente na vida desses pacientes, já que a situação de doença é considerada uma causa externa de estresse, em função da possibilidade de um desequilíbrio na vida do sujeito.

Esse desequilíbrio pode estar relacionado a aspectos físicos e psicológicos (Lipp, Romano, Covolan \& Nery, 1990), como também pode ser resultado da hospitalização, tratamento, mudança na rotina e prejuízos diversos causados pela doença (Marques, 2002). O processo de estresse compreende três fases, identificadas por Selye (1959), que denominou de Síndrome de Adaptação Geral (SAG) o conjunto de reações de adaptação frente ao estressor: reação de alarme, resistência e exaustão. Lipp (1996) acrescenta que tais situações podem ser aquelas que geram medo, excitação ou, até mesmo, alegria.

A princípio, o estresse seria positivo, pois impele o sujeito à ação (Teles, 1993), fenômeno denominado Eustress (França \& Rodrigues, 1996; Jesus, 1999). O estresse negativo foi denominado Distress, pois provoca desordem no organismo e no psiquismo. Diante de situação que exija uma ação, o organismo fica em estado de estresse e, quando a resposta é dada, volta ao equilíbrio. Se o organismo não retornar ao equilíbrio, acontece o estresse negativo. Falcone (1989) ressalta que características pessoais podem levar a diferentes reações e que pessoas, sem recursos emocionais ou físicos, podem desenvolver transtornos diversos. Os fenômenos estressores podem advir do meio externo, como frio, calor, condições de insalubridade, ambiente social, ou do mundo interno, em forma de pensamentos, emoções, angústias, medo, alegria e tristeza (Cataldi, 1999).

Para Selye (1959), a resposta inicial frente a um agente nocivo seria a reação de alarme, na qual o indivíduo sente-se como em estado de choque. No caso de o indivíduo ficar em exposição contínua a qualquer agente nocivo, por tempo indeterminado, pode haver risco de morte. Na segunda fase, de resistência ou adaptação, o indivíduo tenta se adaptar e restabelecer o equilíbrio (Lipp, 1996), utilizando a energia adaptativa de reserva. Após uma exposição mais prolongada a um agente estressante, inicia-se a fase de exaustão, cujos sintomas são semelhantes aos da primeira fase.

Lipp (2000), ao desenvolver o Inventário de Sintomas de Estresse para Adultos de Lipp - ISSL, identificou, ainda, uma quarta fase, denominada quase-exaustão, caracterizada pela dificuldade de adaptação. Segundo essa autora, a reação hormonal desencadeia uma série de reações físicas e emocionais e, assim, de acordo com essa teoria, o surgimento de doenças está vinculado aos sistemas nervoso, endócrino e imunológico. Porém, como ressalta Arantes e Vieira (2002), a predisposição individual deve ser considerada para que ocorra o estabelecimento da doença.

Holmes e Rahe (1967) relacionaram problemas de saúde a mudanças significativas na vida. Sendo assim, quanto maior o número de mudanças ocorridas no espaço de um ano da vida do indivíduo, menor fica a energia adaptativa para enfrentar os estressores. Portanto, aumenta a probabilidade de ocorrerem problemas de saúde.

Para entender as manifestações decorrentes de uma vivência estressante, é preciso conceituar o estresse como sendo um processo, e não como uma reação única. Nesse sentido, Lipp (1998) ressalta que é fundamental a realização de um diagnóstico adequado do quadro de estresse, o que não é fácil, pois muitos sintomas de estresse podem, também, constituírem-se em sintomas de outras doenças.

A partir do exposto acima, o presente artigo relata um estudo cujos objetivos foram: (1) investigar a presença, fase e tipo de resposta (física ou psicológica) de estresse em pacientes com GPAA; (2) avaliar a relação entre o número de colírios utilizados para tratamento e a presença de estresse; e (3) conhecer a percepção de pacientes em relação ao glaucoma e tratamento.

\section{Método}

\section{Participantes}

A pesquisa foi realizada com 102 pacientes diagnosticados com GPAA, maiores de 18 anos, de ambos os sexos, atendidos no Ambulatório de Oftalmologia do Hospital das Clínicas da Faculdade de Medicina da USP, no período de agosto/2006 a setembro/2006.

Para a composição da amostra de conveniência, foram selecionados pacientes que atendiam ao perfil acima e compareceram à consulta de acompanhamento no ambulatório. Foram excluídos da amostra pacientes que não atendiam ao perfil descrito, bem como pacientes que apresentavam quadros prévios de transtornos mentais.

O trabalho foi aprovado pelo Comitê de Ética (Protocolo de Pesquisa $n^{\circ}$ 919/06 da CapPesq) e os pacientes assinaram um Termo de Consentimento Livre e Esclarecido, obedecendo à resolução do Conselho Nacional de Saúde do Ministério da Saúde (CNS 196/96).

\section{Instrumentos}

Foi utilizado um roteiro temático com questões sobre dados sociodemográficos e percepção da doença, e, também, o ISSL (Lipp, 2000).

O ISSL visa identificar os sintomas de estresse, tipos de sintomas (físico ou psicológico) e a fase de estresse (alerta, resistência, quase-exaustão e exaustão). Ele é composto de três quadros que se referem a sintomas típicos encontrados em cada fase do estresse, sendo cada quadro subdividido em 
sintomas físicos e psicológicos. O Quadro 1 corresponde à fase de alerta, o Quadro 2, à fase de resistência e quase-exaustão e o Quadro 3, à fase de exaustão.

\section{Procedimento}

Trata-se de uma pesquisa transversal e quantitativa. A aplicação do questionário foi realizada no Ambulatório de Oftalmologia do Hospital das Clínicas da Faculdade de Medicina da USP. Os pacientes diagnosticados com GPAA e maiores de 18 anos, que passavam em consulta de rotina, foram escolhidos aleatoriamente e convidados a participar da pesquisa. $\mathrm{O}$ questionário foi aplicado individualmente pela pesquisadora, numa sala do ambulatório de oftalmologia, a todos que aceitaram participar da pesquisa.

\section{Análise de dados}

Para análise dos dados, os pacientes foram divididos em dois grupos: Grupo A - composto por 51 pacientes com GPAA, com uso de um ou dois tipos diferentes de colírios para tratamento do glaucoma e Grupo B - composto por 51 pacientes com GPAA, com uso de mais de dois tipos diferentes de colírios para tratamento de glaucoma. Os dados foram digitados em planilha do Microsoft Excel e analisados pelo Software SPSS para Windows versão 12.0.

Foram estabelecidas as relações bivariadas entre 32 variáveis qualitativas e o estresse. A associação entre variáveis qualitativas foi analisada pelo Teste do Qui-quadrado ou Teste Exato de Fisher ou Generalização do Teste Exato de Fisher, quando apropriado. Para as variáveis estatisticamente significantes foram calculadas a Razão de Chances (Odds Ratio), seguido do Intervalo com 95\% de Confiança ( $\mathrm{IC}_{95 \%}$ ) e nível de significância $\alpha=5 \%$.

\section{Resultados}

A Tabela 1 mostra a caracterização sociodemográfica da população investigada e a Tabela 2 apresenta dados sobre a percepção da doença e do tratamento. A maioria dos pacientes $(64,7 \%)$ tem mais de 65 anos e se trata há menos de 10 anos $(56,9 \%)$. A maioria dos pacientes $(79,4 \%)$ acredita que pode ficar cego, 71,6\% acredita que o glaucoma não tem cura e $45,1 \%$ acredita que glaucoma tem tratamento. A maioria $(76,5 \%)$ não tem ajuda para instilar, nem para lembrá-la de instilar os colírios $(88,2 \%)$.

Quanto ao acesso aos colírios, 74,5\% dependem do serviço gratuito da farmácia do hospital e, desses, 24,5\% compram os medicamentos quando faltam na farmácia do hospital.

Conforme indicado na Figura 1, o ISSL mostrou que a maioria dos pacientes $(65,7 \%)$ apresentou estresse, sendo $23,9 \%$ na fase de alerta, $67,1 \%$ na fase de resistência, e $9 \%$, na fase de exaustão. Nenhum paciente se situou na fase de quase-exaustão. Quanto aos sintomas predominantes, 56,7\% apresentara sintomas psicológicos, $29,9 \%$, sintomas físicos e $13,4 \%$, ambos.
Tabela 1. Caracterização sociodemográfica dos 102 pacientes com GPAA

\begin{tabular}{ll}
\hline \multicolumn{1}{c}{ VARIÁVEIS } & n (\%) \\
\hline Sexo & $44(43,1)$ \\
Homens & $58(56,9)$ \\
Mulheres & $67,2 \pm 11,7$ \\
Idade Média \pm d.p. & $6(5,9)$ \\
$35 \vdash-144$ & $11(10,8)$ \\
$45 \vdash-154$ & $19(18,6)$ \\
$55 \vdash-164$ & $37(36,3)$ \\
$65 \vdash-174$ & $29(28,4)$ \\
$\geq 75$ years & \\
Escolaridade & $12(11,8)$ \\
Analfabeto & $19(18,6)$ \\
I grau completo & $60(58,8)$ \\
I grau incompleto & $7(6,9)$ \\
II grau completo & $4(3,9)$ \\
III grau completo &
\end{tabular}

Estado civil

Solteiro (a)

$12(11,8)$

Casado (a)

$56(54,9)$

Divorciado (a)

$11(10,8)$

Viúvo (a)

$23(22,5)$

$\mathbf{N}^{0}$ Filhos Média \pm d.p.

$3,0 \pm 2,34$

\section{Renda Familiar}

$\begin{array}{ll}0 \text { - } 5 \text { salários } & 70(68,6) \\ 5 \text { - } 10 \text { salários } & 16(15,7) \\ 10 \vdash 15 \text { salários } & 1(1,0) \\ >15 \text { salários } & 1(1,0)\end{array}$

Não declarado

Tem outra doença crônica?
Não
$27(26,5)$
Sim
$75(73,5)$

Está trabalhando?

Sim

$28(27,5)$

Não

$74(72,5)$

Fez alguma cirurgia de glaucoma?

Não

$77(75,5)$

$\operatorname{Sim}$

$25(24,5)$ 
Tabela 2. Percepção sobre doença e tratamento dos 102 pacientes com GPAA.

\begin{tabular}{ll}
\hline \multicolumn{1}{c}{ VARIÁVEIS } & n (\%) \\
\hline Quanto tempo trata glaucoma? & \\
$<10$ anos & $58(56,9)$ \\
$\geq 10$ anos & $44(43,1)$ \\
Quando foi a última consulta? & \\
$<3$ meses & $21(20,6)$ \\
$3 \vdash 6$ meses & $20(19,6)$ \\
6 F 12 meses & $52(51,0)$ \\
$\geq 12$ meses & $8(7,8)$ \\
Especificado & $1(1,0)$
\end{tabular}

Glaucoma mudou sua vida?

Não

Sim

Acredita que glaucoma tem cura?

Não sabe

Tem cura

Não tem cura

$27(26,5)$

Religiosidade (Deus pode curar)

$12(11,8)$

Não tem cura, mas tem tratamento

$46(45,1)$

O que acontece se não tratar?

Fica cego

$81(79,4)$

Não acontece nada

$2(2,0)$

Não sabe

$19(18,6)$

Alguém ajuda a lembrar de pingar o colírio?

Sim

Não

Paciente tem ajuda ao instilar?

Às vezes

Nunca

$78(76,5)$

Sempre

Tem outra doença crônica?

Não

Sim

$75(73,5)$

Veio acompanhado na consulta?

Sim

Não

$68(66,7)$

Como adquire o(s) medicamento(s)?

$\mathrm{Na}$ farmácia do HC ou compra

Compra

$29(28,4)$

Exclusivamente na farmácia do HC

$47(46,1)$

Não especificou
A Figura 2 mostra que não houve correlação estatisticamente significante entre idade e presença de estresse $(p=0,778)$. A Figura 3 revela que não foi encontrada correlação estatisticamente significante entre o número de colírios utilizados (se os pacientes usavam apenas um colírio ou mais de um colírio) e o estresse $(\mathrm{p}=0,328 ; \mathrm{OR}=1,731$; $\mathrm{IC}=0,572-5,328)$.

A Tabela 3 apresenta 18 variáveis qualitativas, destacadas com o objetivo de ilustrar o cálculo da análise bivariada. $\mathrm{Na}$ avaliação do estresse, foram encontradas três variáveis estatisticamente significantes:

1. "Quanto tempo trata o glaucoma?" $(p=0,010)$. A chance de estresse em pacientes que tratam o glaucoma há menos de 10 anos é inferior à chance de estresse em pacientes que tratam o glaucoma há 10 anos ou mais $(\mathrm{OR}=0,316$; $\mathrm{IC}=0,129-0,776)$;

2. "Glaucoma dificultou sua vida diária?" $(p=0,02)$. A chance de estresse em pacientes com dificuldades na vida diária geradas pelo glaucoma é inferior à chance de estresse em pacientes sem tais dificuldades $(\mathrm{OR}=0,240$; $\mathrm{IC}=0,095-0,605)$;

3."Tem dificuldade em pingar o colírio?” $(p=0,004)$. A chance de estresse em pacientes que não têm dificuldade em pingar o colírio é inferior à chance de estresse em pacientes que têm dificuldade em pingar o colírio $(\mathrm{OR}=0,203$; $\mathrm{IC}=0,064-0,644)$.

A Tabela 4 mostra as variáveis incluídas no modelo multivariado. A regressão logística com as variáveis estatisticamente significantes no modelo bivariado mostrou que as variáveis acima mantinham significância estatística e independentemente associada ao estresse.

\section{Discussão}

Embora o GPAA constitua um grave problema de saúde pública no Brasil e no mundo, existem poucos trabalhos dedicados ao estudo da presença de estresse entre portadores do mesmo. Dados do estudo realizado revelaram que a maioria dos pacientes tem acima de 65 anos (64,7\%), são aposentados $(72,5 \%)$, tem renda de até cinco salários mínimos $(68,6 \%)$ e $73,5 \%$ deles declararam serem portadores de alguma outra doença crônica.

A maioria dos pacientes $(79,4 \%)$ acredita que pode ficar cego e, de fato, o glaucoma é uma das maiores causas de cegueira no mundo (Susanna Jr. \& Weinreb, 2005). Quanto à percepção sobre a cura, 24,5\% acreditam que o glaucoma tem cura, e, desses, $11,7 \%$ acreditam em cura através da religião.

Sobre o tratamento, $45,1 \%$ acreditam que glaucoma tem tratamento. Esses dados mostram que, em sua maioria, os pacientes dispõem de informações adequadas sobre a doença e tratamento. No entanto, não é possível afirmar se essas informações se revertem em benefício direto no enfrentamento da doença ou na adesão ao tratamento.

O Hospital das Clínicas de São Paulo atende pacientes de baixa renda e quadros oftalmológicos mais graves, o que pode influenciar os resultados deste estudo. A dificuldade de acesso aos medicamentos, por questões financeiras, segundo Silva e cols. (2002), pode ser um fator de estresse entre esses pa- 


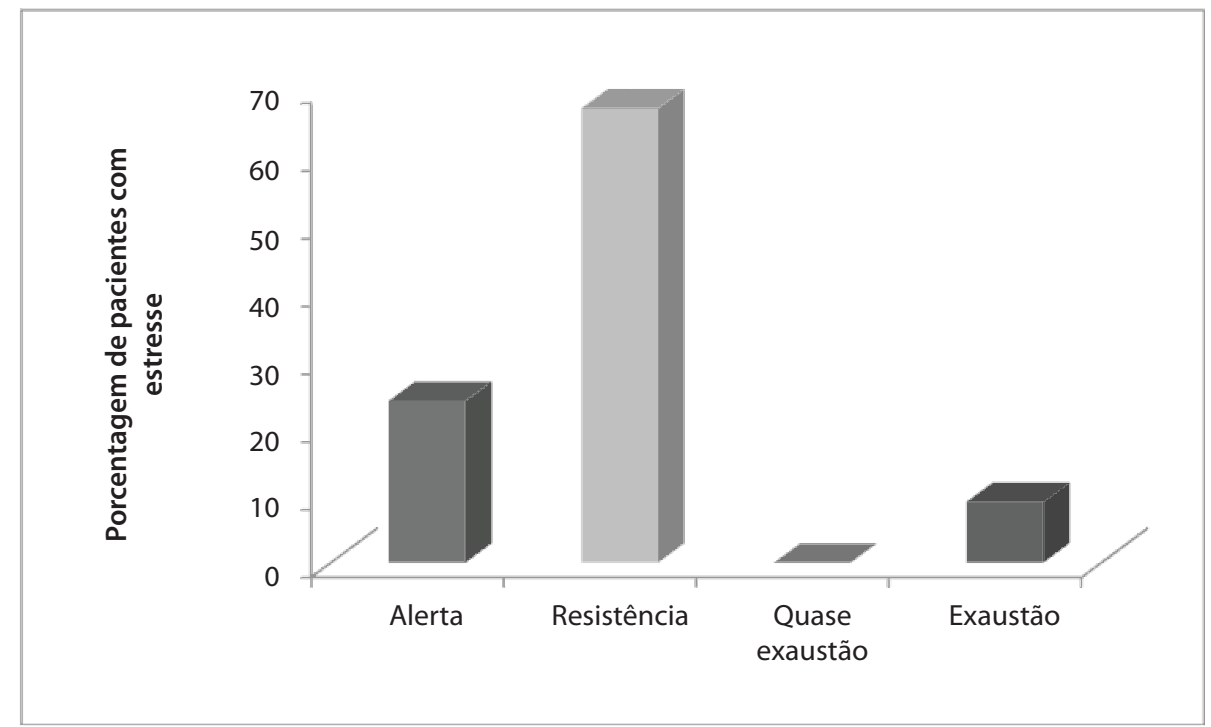

Figura 1. Resultados da fase de estresse entre 102 pacientes com GPAA.

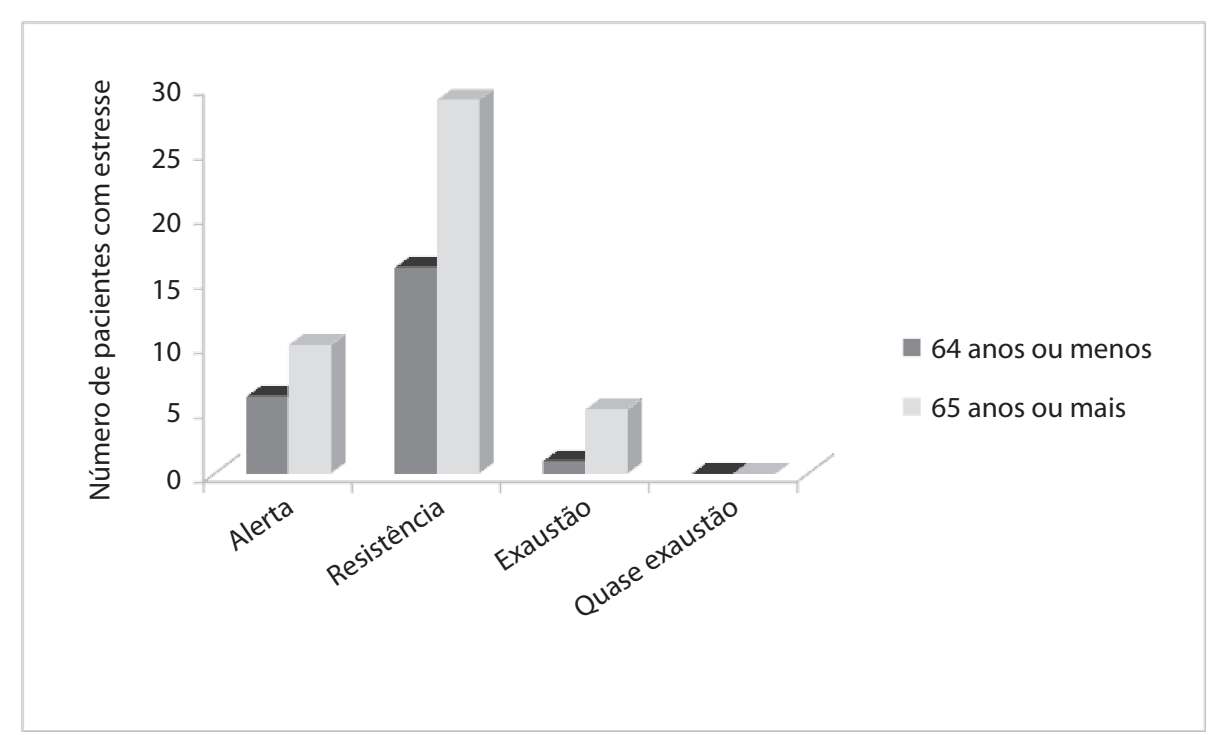

Figura 2. Relação entre idade e fase de estresse entre 102 pacientes com GPAA

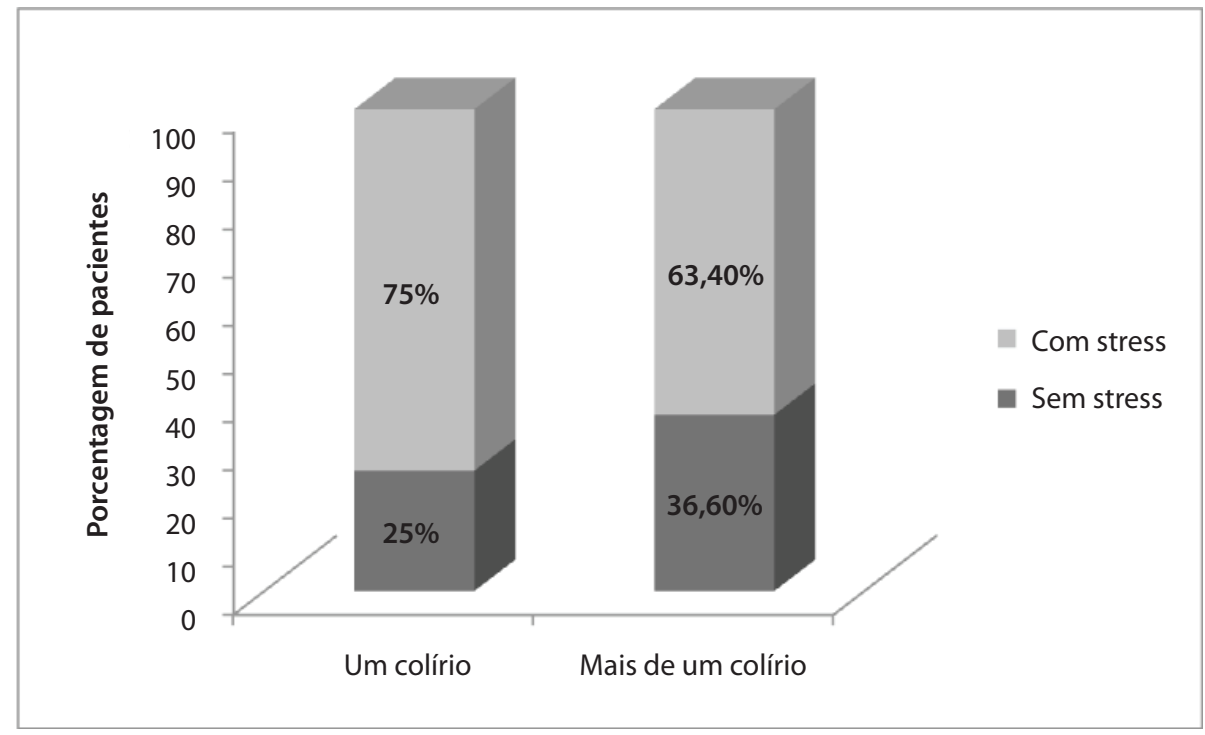

Figura 3. Relação entre número de colírios e presença de estresse entre 102 pacientes com GPAA. 
Tabela 3. Análise bivariada do estresse nos 102 pacientes com GPAA.

\begin{tabular}{|c|c|c|c|}
\hline & \multicolumn{3}{|c|}{ ESTRESSE } \\
\hline & p-valor* & OR $95 \% * *$ & IC*** \\
\hline Sexo & 0,423 & 1,399 & $0,614-3,187$ \\
\hline Idade (anos) & 0,778 & 0,885 & $0,378-2,072$ \\
\hline Grau instrução & 0,090 & 2,125 & $0,882-5,118$ \\
\hline Quanto tempo trata o glaucoma? & $0,010^{*}$ & 0,316 & $0,129-0,776$ \\
\hline Quando foi a ultima consulta? & 0,682 & 0,813 & $0,301-2,197$ \\
\hline Fez cirurgia relacionada ao glaucoma? & 0,779 & 0,871 & $0,333-2,280$ \\
\hline Glaucoma dificultou sua vida diária? & $0,020^{*}$ & 0,240 & $0,095-0,605$ \\
\hline Se não tratar o glaucoma pode ficar cego? & 0,664 & 0,955 & $0,166-5,487$ \\
\hline Há quanto tempo trata glaucoma com colírio? & 0,060 & 0,427 & $0,174-1,048$ \\
\hline Quantas vezes ao dia pinga colírio? & 0,744 & 0,691 & $0,171-2,790$ \\
\hline Esquece de pingar o colírio? & 0,192 & 0,565 & $0,239-1,337$ \\
\hline Interrompeu o tratamento em algum momento? & 0,149 & 0,454 & $0,153-1,349$ \\
\hline Tem dificuldades em pingar o colírio? & $0,004 *$ & 0,203 & $0,064-0,644$ \\
\hline Alguém lembra ou ajuda pinga o colírio? & 0,211 & 0,345 & $0,071-1,673$ \\
\hline Tem dúvida no que o médico explicou? & 0,534 & 0,534 & $0,137-2,084$ \\
\hline Falta às consultas agendadas? & 0,712 & 0,616 & $0,118-3,226$ \\
\hline Paciente conhece a prescrição? & 0,788 & 0,862 & $0,292-2,542$ \\
\hline Tempo de instilação entre os colírios & 0,878 & 1,091 & $0,359-3,315$ \\
\hline
\end{tabular}

Nota: $* \mathrm{p}<0,05 ; * *$ OR: Odds ratio; *** IC: Intervalo de confiança.

Tabela 4. Análise Multivariada das variáveis cujo p-valor foi significante na análise bivariada.

\begin{tabular}{lccc}
\hline & \multicolumn{3}{c}{ ESTRESSE } \\
\cline { 2 - 4 } & p-valor* & OR** $^{* *}$ & IC $^{* * *}$ \\
\hline Dificuldades em pingar colírio? & 0,004 & 3,698 & {$[1,114-12,274]$} \\
Glaucoma dificultou sua vida diária? & 0,020 & 0,277 & {$[0,106-0,724]$} \\
Quanto tempo trata o glaucoma? & 0,010 & 1,966 & {$[0,745-5,188]$} \\
\hline
\end{tabular}

Nota: $* \mathrm{p}<0,05 ; * *$ OR: Odds ratio; *** IC: Intervalo de confiança.

cientes. Nesse sentido, é importante ressaltar que, no referido hospital, existe um programa de entrega gratuita dos colírios e a maioria dos pacientes $(74,5 \%)$ depende desse serviço.

Em relação ao tratamento, em quadros de maior progressão da doença, o número de colírios usados é maior, tornando o esquema terapêutico mais complexo e de pior adesão. A hipótese era que maior número de colírios poderia se relacionar com estresse, mas os dados não mostraram correlação estaticamente significante entre número de colírios e estresse $(\mathrm{p}=0,328)$.

Problemas relacionados ao apoio familiar podem se mostrar logicamente envolvidos no quadro de estresse dos pacientes, considerando que a idade média dos mesmos foi de 67 anos e que a maioria $(73,5 \%)$ apresentava outra doen- ça crônica associada. O trabalho de Winfield e cols. (1990) mostrou que $62 \%$ dos pacientes autoadministrava o colírio e referia poder se beneficiar da ajuda de outras pessoas para instilar ou lembrar de instilar os colírios. Entretanto, não foi observada correlação estatisticamente significante entre a presença de alguém para lembrar ou pingar os colírios $(\mathrm{p}=$ 0,211 ) ou quanto à presença de um acompanhante durante as consultas $(p=0,582)$ em relação ao estresse.

Os resultados do ISSL (Lipp, 2000) mostraram que 65,7\% dos pacientes apresentam algum tipo de estresse, sendo que a maioria desses pacientes está na fase de resistência $(67,1 \%)$. Essa predominância aponta uma tentativa de adaptação ao estresse. Encontram-se 9\% dos pacientes na fase de exaustão, 
mostrando a vivência contínua de estresse, com prováveis prejuízos físicos importantes (Jesus, 1999).

Esses dados levam a entender que grande parte dos pacientes com GPAA, participantes do estudo, está vivenciando uma desordem física ou psíquica, e trabalhos como os de Jampel e cols. (2007) e Mabuchi e cols. (2008), bem como de Spinelli e cols. (1998), corroboram essa constatação, pois encontraram significativa prevalência de ansiedade e depressão (alterações de humor) entre pessoas com GPAA, especialmente entre pacientes recentemente diagnosticados ou que apresentaram piora da função visual, referida pelos mesmos.

Embora nesta investigação não tenham sido relacionados os dados de estresse com os níveis pressóricos oculares dos pacientes, pode-se destacar o interessante trabalho de Kaluza, Strempel e Maurer (1996). Esses autores investigaram a influência do estresse na elevação da PIO e concluíram que, mediante indução psicofisiológica, os níveis de PIO podem se elevar; entretanto, treinos de relaxamento não apresentam influência na PIO. Tal pesquisa parece nos fazer perceber que a relação entre stress e elevação da PIO pode ser complexa e que o uso de técnicas de relaxamento pode não ter influência nesse processo.

O tempo de tratamento mostrou-se uma variável importante, pois foi estatisticamente significante e independentemente associada ao estresse $(\mathrm{p}=0,01 ; \mathrm{OR}=0,316 ; \mathrm{IC}=0,129$ - 0,776). A maior presença de estresse em pacientes que se tratam há mais tempo pode estar relacionada, também, com o fato de que a maioria se encontra na fase de resistência $(67,1 \%)$, ou seja, o fator tempo é um fator independente na tentativa de adaptação dos sujeitos ao estresse.

Demailly e cols. (1989) observaram, ainda, que, além de a adesão ser maior no início do tratamento, a presença de transtornos de ansiedade é mais significativa entre pacientes glaucomatosos crônicos, o que leva a refletir que, ao longo do tempo, os pacientes parecem ter mais complicações de ordem emocional, incluindo estresse. Haverá, consequentemente, interferência na forma como eles lidam com sua doença.

Outra variável estatisticamente significante e independentemente associada ao estresse foi se o "glaucoma dificultou a vida diária" dos pacientes entrevistados $(\mathrm{p}=0,02)$. Esse resultado parece lógico e indica, não uma relação de causa e efeito, mas uma correlação, ou seja, não é possível dizer se é o estresse que causa dificuldades na vida diária ou se essas dificuldades causam estresse. Pacientes que referiram dificuldades em pingar colírios apresentaram mais estresse ( $\mathrm{p}=0,004 ; \mathrm{OR}=0,203 ; \mathrm{IC}=0,064-0,644)$, e, da mesma forma, observou-se uma correlação estatística importante e não uma relação de causa e efeito. Esses dados podem reforçar os encontrados por Demailly e cols. (1989), Lim e cols. (2007) e Spinelli e cols. (1998), que relatam mudanças na vida diária como um fator significativo para esses pacientes.

Mediante esse quadro, é razoável compreender que portadores de GPAA podem apresentar alteração na qualidade de vida, objeto de estudo de Perfetti e cols. (1998). Eles concluíram que o diagnóstico de GPAA piora a qualidade de vida desses pacientes em $1 / 3$ e que aqueles mais esclarecidos a respeito da doença e do tratamento tendem a apresentar melhor adesão e melhor qualidade de vida.

A fala de uma paciente de 81 anos pode ilustrar os achados deste estudo: "A pessoa sem vista (por causa do Glaucoma) não é nada" (sic), pois explicita o sofrimento do paciente diante de uma doença grave que pode levar a uma perda fundamental e irreversível. Essa fala sugere que, além de apresentar algum tipo de estresse, o que foi observado em $65 \%$ dos participantes aqui entrevistados, os pacientes têm que enfrentar mudanças na sua autopercepção, pois passam a se perceber como pessoas com limitações e, por isso, sem valor.

Sendo assim, os pacientes têm que conviver não somente com uma doença incurável (mas que pode ser controlada), mas também com as consequências orgânicas e emocionais por ela provocadas. Os sintomas de estresse, tão frequentes nessa população, parecem contribuir negativamente, fazendo com que pacientes e seus familiares sejam ainda mais exigidos diante do desafio do enfrentamento do glaucoma, investimento em autocuidado e construção de uma melhor qualidade de vida.

Diante desse quadro, considera-se fundamental a atuação de uma equipe multiprofissional que se disponha a realizar um trabalho articulado, capaz de oferecer uma "escuta" e de lançar um "olhar" para o sujeito que, perante a perda visual, vê-se diante do questionamento de sua identidade, segundo o qual "não ver" equivale a "não ser nada". Nesse trabalho multiprofissional, o desafio de "ver além da doença", ou seja, "enxergar o sujeito que vive com glaucoma" é a base de um trabalho calcado na Ética.

Em conclusão, o estresse está presente em mais da metade dos pacientes entrevistados em um serviço público de referência, de alta complexidade, para atendimento de pessoas com GPAA, constituindo-se em um aspecto fundamental para a compreensão da forma como pacientes lidam com o glaucoma. As variáveis 'tempo de tratamento do glaucoma', 'dificuldades na vida diária devidas ao glaucoma' e 'dificuldades em instilar o colírio' apresentaram-se independentemente associadas ao estresse e devem ser enfaticamente consideradas no planejamento de programas de intervenção sobre adesão e qualidade de vida em serviços de saúde que atendem pacientes com GPAA.

\section{Referências}

Arantes, M. A. C., \& Vieira M. J. F. (2002). Estresse (2ª ed.). São Paulo: Casa do Psicólogo.

Azura-Blanco, A. (2002). Handbook of glaucoma. United Kingdom: Martin Dunitz.

Balkrishnan, R., Bond, J. B., Byerly, W. G., Camanho, F. T., \& Anderson, R. T. (2003). Medication-related predictors of healthrelated quality of life in glaucoma patients enrolled in a medicare health maintenance organization. American Journal Geriatric Pharmacy, 1, 75-81.

Cataldi, M. J. G. (1999). O estresse no meio ambiente do trabalho. Dissertação de Mestrado, Pontifícia Universidade Católica de São Paulo, São Paulo.

Demailly, P., Zoute, C., \& Castro, D. (1989). Personalities and chronic glaucoma. Journal Français d'Ophtalmologie, 12, 595-601.

Deokule, S., Sadiq, S., \& Shah, S. (2004). Chronic open angle glaucoma: Patient awareness of the nature of the disease, topical medication, compliance and the prevalence of systemic symptoms. Ophthalmic and Physiological Optics, 1, 9-15. 
Falcone, E. M. O. A. (1989). A eficácia do tratamento em grupo da ansiedade social. Dissertação de Mestrado, Pontifícia Universidade Católica do Rio de Janeiro, Rio de Janeiro.

Flammer, S. (2001). Glaucoma. Germany: Hans Huber.

França, A. C. L., \& Rodrigues, A. L. (1996). Estresse e trabalho: guia básico com abordagem psicossomática. São Paulo: Atlas.

Gabelt, A., \& Kaufman, P. L. (2005). Changes in aqueous humor dynamius with age and glaucoma. Progress in Retinal and Eye Research, 1, 612-637.

Holmes, T. H., \& Rahe, R. K. (1967). The social readjustment rating scale. Journal of Psychosomatic Research, 11, 213-218.

Jampel, H. D., Frick, K. D., Janz, N. K., Wren, P. A., Musch, D. C., Rimal, R., \& Lichter, P. R. (2007). Depression and mood indicators in newly diagnosed glaucoma patients. American Journal of Ophthalmology, 144, 238-244.

Jesus, S. N. (1999). Como prevenir e resolver o estresse dos professores e a indisciplina dos alunos. Porto: Asa.

Kaluza, G., Strempel, I., \& Maurer, H. (1996). Stress reactivity of intraocular pressure after relaxation training in open-angle glaucoma patients. Journal of Behavioral Medicine, 19, 587-598.

Lim M. C., Shiba D. R., Clark I. J., Kim D. Y., Styles D. E., Brandt J. D., Watnik M. R., \& Barthelow I. J. (2007). Personality type of the glaucoma patient. Journal of Glaucoma, 16, 649-654.

Lipp, M. E. N. (1996). Pesquisa sobre estresse no Brasil: saúde, ocupações e grupos de risco. Campinas: Papirus.

Lipp, M. E. N. (1998). O estresse está dentro de você. São Paulo: Contexto.

Lipp, M. E. N. (2000). Manual do Inventário de Sintomas de Estresse para Adultos de Lipp (INSS). São Paulo: Casa do Psicólogo.

Lipp, M. E. N., Romano, A. S. P. F., Covolan, M. A., \& Nery, M. J. G. S. (1990). Como enfrentar o estresse ( $5^{\mathrm{a}} \mathrm{ed}$.). Campinas: Ícone.

Mabuchi, F., Yoshimura, K., Kashiwagi, K., Shioe, K., Yamagata, Z., Kanba, S., Iijima, H., \& Tsukahara, S. (2008). High prevalence of anxiety and depression in patients with primary openangle glaucoma. Journal of Glaucoma, 17, 552-557.

Marques, A. (2002). Câncer e estresse: um estudo sobre crianças em tratamento quimioterápico. Psicologia Hospitalar, 2, 1-15.
Perfetti, S., Varotto, A., Massagrandi, S., Pagliani, F., \& Bonomi, L. (1998). Glaucoma and quality of the life. Acta Ophthalmologica Scandinavica, 76, 52-54.

Selye, H. (1959). Estresse: a tensão da vida (2a ed.). São Paulo: Ibrasa.

Silva, L. M. S., Vasconcellos, J. P. C., Temporim, E. R., Costa, V. P., \& Kara- José, M. (2002). Tratamento clínico do glaucoma em um hospital universitário: custo mensal e impacto na renda familiar. Arquivos Brasileiros de Oftalmologia, 1, 1-3.

Spaeth, G., Walt, J., \& Kenner, J. (2006). Evoluation of quality of life for patientes with glaucoma. American Journal of Ophthalmology, 1, 785-797.

Spinelli, D., Faroni, E., \& Castellini, G. (1998). The "personality" of the glaucomatous patient: Preliminary results. Acta Ophthalmologica Scandinavica, 76, 53-54.

Susanna Jr., R., \& Weinreb, R. N. (2005). Glaucoma: perguntas e respostas. Rio de Janeiro: Cultura Médica.

Teles, M. L. S. (1993). O que é estresse. São Paulo: Brasiliense.

Vaidergorn, R. G., Malta, R., Borges A. S., Menezes, M. D. R., Trindade, E. S., \& Malta, J. B. N. S. (2003). Técnica da instilação de colírios em portadores de glaucoma crônico. Arquivos Brasileiros de Oftalmologia, 1, 865-869.

Wilensky, J. T. (1996). The role of brimonidine in the treatment of open angle glaucoma. Survey of Ophthalmology, 1, 3-7.

Winfield, A. J., Jessiman, D., Williams, A., \& Esakowitz, L. (1990). A study of the causes of non-compliance by patients prescribed eyedrops. British Journal of Ophthalmology, 74, 477-480.

Recebido em 19.04.08

Primeira decisão editorial em 27.07.09

Versão final em 27.08.10

Aceito em 27.08.10 